PROCEEDINGS OF THE

AMERICAN MATHEMATICAL SOCIETY

Volume 134, Number 3, Pages 689-695

S 0002-9939(05)08338-3

Article electronically published on October 17, 2005

\title{
EQUICOMPACT SETS OF OPERATORS DEFINED ON BANACH SPACES
}

\author{
E. SERRANO, C. PIÑEIRO, AND J. M. DELGADO \\ (Communicated by Jonathan M. Borwein)
}

\begin{abstract}
Let $X$ and $Y$ be Banach spaces. We say that a set $\mathcal{M} \subset \mathcal{K}(X, Y)$ $(\mathcal{K}(X, Y)$ denotes the space of all compact operators from $X$ into $Y)$ is equicompact if there exists a null sequence $\left(x_{n}^{*}\right)_{n}$ in $X^{*}$ such that $\|T x\| \leq \sup _{n}\left|x_{n}^{*}(x)\right|$ for all $x \in X$ and all $T \in \mathcal{M}$. It is easy to show that collectively compactness and equicompactness are dual concepts in the following sense: $\mathcal{M}$ is equicompact iff $\mathcal{M}^{*}=\left\{T^{*}: T \in \mathcal{N}\right\}$ is collectively compact. We study some properties of equicompact sets and, among other results, we prove: 1) a set $\mathcal{M} \subset \mathcal{K}(X, Y)$ is equicompact iff each bounded sequence $\left(x_{n}\right)_{n}$ in $X$ has a subsequence $\left(x_{k(n)}\right)_{n}$ such that $\left(T x_{k(n)}\right)_{n}$ is a converging sequence uniformly for $T \in \mathcal{M} ; 2)$ if $Y$ does not have finite cotype and $\mathcal{N} \subset \mathcal{K}(X, Y)$ is a maximal equicompact set, then, given $\varepsilon>0$ and a finite set $\left\{x_{1}, \ldots, x_{n}\right\}$ in $X$, there is an operator $S \in \mathcal{M}$ such that $\left\|T x_{i}\right\| \leq(1+\varepsilon)\left\|S x_{i}\right\|$ for $i=1, \ldots, n$ and all $T \in \mathcal{M}$.
\end{abstract}

\section{INTRODUCTION}

Let us consider (real or complex) Banach spaces $X$ and $Y$. As usual $\mathcal{K}(X, Y)$ will denote the vector space of all compact linear maps endowed with the operator norm. We say that a set $\mathcal{N} \subset \mathcal{K}(X, Y)$ is equicompact if there exists a null sequence $\left(x_{n}^{*}\right)_{n}$ in $X^{*}$ so that

$$
\|T x\| \leq \sup _{n}\left|x_{n}^{*}(x)\right|
$$

for all $x \in X$ and all $T \in \mathcal{M}$. We recall that a set $\mathcal{M} \subset \mathcal{K}(X, Y)$ is called collectively compact iff $\bigcup_{T \in \mathcal{M}} T\left(B_{X}\right)$ has compact closure. A standard proof using separations theorems and the well-known fact that a compact set in a Banach space is contained in the closure of the convex hull of a null sequence of the space allows us to state that $\mathcal{M}$ is equicompact iff $\mathcal{M}^{*}=\left\{T^{*} \in \mathcal{K}\left(Y^{*}, X^{*}\right): T \in \mathcal{M}\right\}$ is collectively compact. We study some properties of equicompact sets. Among other results we have proved the following:

(1) A set $\mathcal{M} \subset \mathcal{K}(X, Y)$ is equicompact iff $\mathcal{N}$ satisfies the next property (invoked as property $(P))$ :

$(P) \quad$ "For every bounded sequence $\left(x_{n}\right)_{n}$ in $X$ there exists a subsequence $\left(x_{k(n)}\right)_{n}$ so that $\left(T x_{k(n)}\right)_{n}$ is a converging sequence uniformly for $T \in \mathcal{M}$."

Received by the editors April 20, 2004.

2000 Mathematics Subject Classification. Primary 47B07.

Key words and phrases. Compact operators, equicompact set, collectively compact set.

(C)2005 American Mathematical Society Reverts to public domain 28 years from publication 
(2) Given a null sequence $\left(x_{n}^{*}\right)_{n}$ in $X^{*}$, we will denote by $\mathcal{M}\left(\left(x_{n}^{*}\right)_{n}\right)$ the equicompact set of all compact operators $T \in \mathcal{K}(X, Y)$ satisfying $\|T x\| \leq$ $\sup _{n}\left|x_{n}^{*}(x)\right|$ for all $x \in X$. These sets are absolutely convex and closed in the strong operator topology. If $Y$ does not have finite cotype we prove that, given $\varepsilon>0$ and a finite set $\left\{x_{1}, \ldots, x_{n}\right\}$ in $X$, there exists an operator $Q \in \mathcal{M}\left(\left(x_{n}^{*}\right)_{n}\right)$ so that $\left\|T x_{i}\right\| \leq(1+\varepsilon)\left\|Q x_{i}\right\|$ for $i=1, \ldots, n$ and all $T \in \mathcal{M}\left(\left(x_{n}^{*}\right)_{n}\right)$.

We also have obtained a partial converse of (2): if $\mathcal{M} \subset \mathcal{K}(X, Y)$ is countably compact for the strong operator topology (for short, SOT), then $\mathcal{M}$ is equicompact when it verifies the following property (invoked as property $(F)$ ):

$$
\begin{aligned}
& \text { There exists a positive constant } C \text { such that, for every } \\
& \text { finite set }\left\{x_{1}, \ldots, x_{n}\right\} \subset X \text { there is an operator } Q \text { in the } \\
& \text { closed absolutely convex hull of } \mathcal{M} \text { satisfying }\left\|T x_{i}\right\| \leq \\
& C\left\|Q x_{i}\right\| \text { for } i=1, \ldots, n \text { and all } T \in \mathcal{M} \text {. }
\end{aligned}
$$

Our notation is standard. If $X$ is a Banach space, $B_{X}$ will denote its closed unit ball. If $I$ is an arbitrary index set, we will write $\ell_{a}^{1}(I, X)$ (respectively, $\ell^{\infty}(I, X)$ ) for the Banach space of all absolutely summable (respectively, bounded) $X$-valued functions defined on $I$, endowed with the norm $\|\xi\|=\sum_{i \in I}\|\xi(i)\|$ (respectively, $\|\xi\|=\sup \{\|\xi(i)\|: i \in I\})$ for each $\xi \in \ell_{a}^{1}(I, X)$ (respectively, $\left.\xi \in \ell^{\infty}(I, X)\right)$.

\section{EQUICOMPACT SETS}

If $\mathcal{M} \subset \mathcal{K}(X, Y)$ is bounded, we can consider the continuous linear map $U: \ell_{a}^{1}(\mathcal{M}, X) \longrightarrow Y$ defined by $U(\xi)=\sum_{T \in \mathcal{M}} T(\xi(T))$ for all $\xi \in \ell_{a}^{1}(\mathcal{M}, X)$.

Proposition 2.1. The following statements are equivalent for a set $\mathcal{M} \subset \mathcal{K}(X, Y)$ :

a) $\mathcal{M}$ is collectively compact.

b) The operator $U$ is compact.

Proof. Given $S \in \mathcal{M}$ and $x \in B_{X}$, denote by $\xi_{S, x}$ the element of $\ell_{a}^{1}(\mathcal{M}, X)$ defined by

$$
\xi_{S, x}(T)= \begin{cases}0 & \text { if } T \neq S \\ x & \text { if } T=S .\end{cases}
$$

It is clear that $U\left(\xi_{S, x}\right)=S x$; then $H=U\left\{\xi_{S, x}: S \in \mathcal{M}, x \in B_{X}\right\}=\bigcup_{T \in \mathcal{M}} T\left(B_{X}\right)$. On the other hand, for every $\xi \in B_{\ell_{a}^{1}(\mathcal{M}, X)}$ we have:

$$
U(\xi)=\sum_{T \in \mathcal{M}} T(\xi(T))=\sum_{\substack{T \in \mathcal{M} \\ \xi(T) \neq 0}}\|\xi(T)\| T\left(\frac{\xi(T)}{\|\xi(T)\|}\right) .
$$

Therefore, $U\left(B_{\ell_{a}^{1}(\mathcal{M}, X)}\right) \subset \overline{\mathrm{co}}(H)$. So we have obtained $H \subset U\left(B_{\ell_{a}^{1}(\mathcal{M}, X)}\right) \subset \overline{\mathrm{co}}(H)$ and this concludes the proof.

Now we consider the operator $V: X \longrightarrow \ell^{\infty}(\mathcal{M}, Y)$ defined by $(V x)(T)=T x$ for all $T \in \mathcal{M}$ and $x \in X$. For the proof of the following proposition, notice that the equivalence $a) \Leftrightarrow b$ ) is obvious and $b) \Leftrightarrow c$ ) is straightforward.

Proposition 2.2. The following statements are equivalent for a set $\mathcal{M} \subset \mathcal{K}(X, Y)$ :

a) $\mathcal{M}$ is equicompact.

b) The operator $V$ is compact.

c) $\mathcal{M}$ has property $(P)$. 
Now we are ready to face our main result:

Theorem 2.3. If $\mathcal{N} \subset \mathcal{K}(X, Y)$ is bounded, the following statements are equivalent:

a) $\mathcal{M}$ is collectively compact.

b) $\mathcal{M}^{*}$ has property $(P)$.

Proof. The adjoint map of $U: \ell_{a}^{1}(\mathcal{M}, X) \longrightarrow Y$ is the operator $U^{*}: Y^{*} \longrightarrow \ell^{\infty}\left(\mathcal{M}, X^{*}\right)$ defined by $\left(U^{*} y^{*}\right)(T)=T^{*} y^{*}$, for all $T \in \mathcal{M}$ and $y^{*} \in Y^{*}$. In fact, we have:

$$
\left\langle\xi, U^{*} y^{*}\right\rangle=\left\langle U \xi, y^{*}\right\rangle=\sum_{T \in \mathcal{M}}\left\langle T(\xi(T)), y^{*}\right\rangle=\sum_{T \in \mathcal{M}}\left\langle\xi(T), T^{*} y^{*}\right\rangle
$$

for all $\xi \in \ell_{a}^{1}(\mathcal{M}, X)$ and $y^{*} \in Y^{*}$. A call to Propositions 2.1 and 2.2 concludes the proof.

The next lemma easily yields the dual equivalence

$\mathcal{M}$ has property $(P) \quad \Longleftrightarrow \quad \mathcal{M}^{*}$ is collectively compact.

Lemma 2.4. If $\left(x_{n}^{*}\right)_{n}$ is a null sequence in $X^{*}$ and $\mathcal{M}$ is a subset of $\mathcal{K}(X, Y)$ such that

$$
\|T x\| \leq \sup _{n}\left|x_{n}^{*}(x)\right|
$$

for all $x \in X$ and all $T \in \mathcal{M}$, then

$$
\left\|T^{* *} x^{* *}\right\| \leq \sup _{n}\left|x^{* *}\left(x_{n}^{*}\right)\right|
$$

for all $x^{* *} \in X^{* *}$ and $T \in \mathcal{M}$.

Proof. Given $T \in \mathcal{M}, \varepsilon>0$ and $x^{* *} \in B_{X^{* *}}$, choose $y^{*} \in B_{Y^{*}}$ so that $\left\|T^{* *} x^{* *}\right\|=$ $\left|y^{*}\left(T^{* *} x^{* *}\right)\right|$. By hypothesis, there exists $n_{0} \in \mathbb{N}$ such that $\left\|x_{n}^{*}\right\|<\varepsilon / 4$ for all $n \geq n_{0}$. Now we consider the weak* neighborhood $W=W\left(x_{1}^{*}, \ldots, x_{n_{0}}^{*}, T^{*} y^{*} ; \varepsilon / 2\right)$ of 0 ; there exists $x \in B_{X}$ satisfying $x \in x^{* *}+W$. Then we have:

$$
\begin{aligned}
\left\|T^{* *} x^{* *}\right\| & =\left|\left\langle T^{*} y^{*}, x^{* *}\right\rangle\right| \\
& \leq\left|\left\langle T^{*} y^{*}, x^{* *}\right\rangle-\left\langle T^{*} y^{*}, x\right\rangle\right|+\left|\left\langle T^{*} y^{*}, x\right\rangle\right| \\
& <\frac{\varepsilon}{2}+\sup _{n}\left|\left\langle x_{n}^{*}, x\right\rangle\right| \\
& \leq \frac{\varepsilon}{2}+\sup _{n}\left|\left\langle x_{n}^{*}, x\right\rangle-\left\langle x_{n}^{*}, x^{* *}\right\rangle\right|+\sup _{n}\left|\left\langle x_{n}^{*}, x^{* *}\right\rangle\right| \\
& <\varepsilon+\sup _{n}\left|\left\langle x_{n}^{*}, x^{* *}\right\rangle\right|
\end{aligned}
$$

for all $\varepsilon>0$. Letting $\varepsilon \rightarrow 0$, we obtain $\left\|T^{* *} x^{* *}\right\| \leq \sup _{n}\left|x^{* *}\left(x_{n}^{*}\right)\right|$ for all $x^{* *} \in X^{* *}$ and all $T \in \mathcal{M}$.

Remark 2.5. A set $\mathcal{M} \subset \mathcal{L}(X, Y)$ is sequentially weak-norm equicontinuous (or uniformly completely continuous) if, for every weakly null sequence $\left(x_{n}\right)_{n}$ in $X$, $\lim _{n}\left\|T x_{n}\right\|=0$ uniformly for $T \in \mathcal{M}$. It is obvious that every equicompact set is uniformly completely continuous (for short, u.c.c.). If $X$ does not contain a copy of $\ell^{1}$, then Rosenthal's theorem about $\ell^{1}$ tells us that each bounded sequence in $X$ has a weakly Cauchy subsequence. So, in this case, every u.c.c. set has property $(P)$ and, therefore, it is equicompact. That is to say, if $X \nsupseteq \ell^{1}$, then the following 
statements are equivalent for a bounded set $\mathcal{M} \subset \mathcal{K}(X, Y)$ :

a) $\mathcal{M}$ is equicompact.

b) $\mathcal{M}$ is u.c.c.

c) $\mathcal{M}^{*}$ is collectively compact.

The equivalence stated in Remark 2.5] and [4, theorem 2.2] yields directly the recent Mayoral's theorem [3]:

Theorem (F. Mayoral, 2001). If $X$ does not contain a copy of $\ell^{1}$, a set $\mathcal{M} \subset$ $\mathcal{K}(X, Y)$ is relatively compact iff $\mathcal{M}$ is u.c.c. and, for every $x \in X$, the set $\mathcal{M}(x)=$ $\{T x: T \in \mathcal{M}\}$ is relatively compact in $Y$.

Nevertheless, for an arbitrary Banach space $X$, a u.c.c. set $\mathcal{M} \subset \mathcal{K}(X, Y)$ is equicompact if, in addition, every $\ell^{1}$-sequence $\left(x_{n}\right)_{n}$ in $X$ has a subsequence $\left(x_{k(n)}\right)_{n}$ such that $\left(T x_{k(n)}\right)_{n}$ is uniformly convergent for $T \in \mathcal{M}$.

\section{Dominated Sets OF OPERATORS}

The simplest examples of equicompact sets are the sets dominated by a compact operator, that is to say, the sets for which there exists an operator $S \in \mathcal{K}(X, Y)$ such that $\|T x\| \leq\|S x\|$ for all $x \in X$ and all $T \in \mathcal{M}$. We are going to prove that a maximal equicompact set $\mathcal{M}$ is dominated by an operator $Q_{H} \in \mathcal{M}$ on every finite set $H=\left\{x_{1}, \ldots, x_{n}\right\} \subset X$. But a more general class of sets $\mathcal{M} \subset \mathcal{L}(X, Y)$ enjoys this property. That is why we now consider the class of $(Z, S)$-dominated sets.

Given a Banach space $Z$ and a linear map $S: X \longrightarrow Z$, we say that a set $\mathcal{M} \subset \mathcal{L}(X, Y)$ is $(Z, S)$-dominated if $\|T x\| \leq\|S x\|$ for all $x \in X$ and all $T \in \mathcal{M}$.

\section{Examples.}

(1) If $\mathcal{M} \subset \mathcal{K}(X, Y)$ is an equicompact set satisfying $\|T x\| \leq \sup _{n}\left|x_{n}^{*}(x)\right|$ for all $x \in X$ and all $T \in \mathcal{M}$, with $\left(x_{n}^{*}\right)_{n}$ a null sequence in $X^{*}$, consider the map $S: X \longrightarrow c_{0}$ defined by $S x=\left(x_{n}^{*}(x)\right)_{n}$. Then, $\mathcal{M}$ is $\left(c_{0}, S\right)$-dominated.

(2) Let $\Pi_{p}(X, Y)$ be the space of $p$-summing operators from $X$ into $Y$ endowed with the norm $\pi_{p}(T)=\sup \left\{\left(\sum_{n}\left\|T x_{n}\right\|^{p}\right)^{1 / p}:\left(x_{n}\right)_{n} \in B_{\ell_{w}^{p}(X)}\right\}$, where $\ell_{w}^{p}(X)$ is the Banach space of the weakly $p$-summable sequences in $X$. A set $\mathcal{M} \subset \Pi_{p}(X, Y)$ is said to be uniformly $p$-dominated if there exists a positive Radon measure $\mu$ on $B_{X^{*}}$ such that

$$
\|T x\|^{p} \leq \int_{B_{X^{*}}}\left|x^{*}(x)\right|^{p} d \mu\left(x^{*}\right)
$$

for all $x \in X$ and all $T \in \mathcal{M}$. Put $Z=L^{p}\left(\mu, B_{X^{*}}\right)$ and define $S: X \longrightarrow Z$ by $(S x)\left(x^{*}\right)=x^{*}(x)$ for all $x^{*} \in B_{X^{*}}$ and all $x \in X$. Then every uniformly $p$-dominated set is $(Z, S)$-dominated.

If $X$ and $Y$ are Banach spaces, we will denote by $\mathcal{M}(Z, S)$ the set of all operators $T \in \mathcal{L}(X, Y)$ satisfying $\|T x\| \leq\|S x\|$ for all $x \in X$. Note that $\mathcal{M}(Z, S)$ is absolutely convex and closed in $\mathcal{K}(X, Y)$ for the SOT topology. The next theorem shows that $\mathcal{M}=\mathcal{M}(Z, S)$ is dominated by an operator $Q \in \mathcal{M}$ on every finite set $\left\{x_{1}, \ldots, x_{n}\right\} \subset$ $X$ when $Y$ does not have finite cotype. 
Theorem 3.1. Let $Y$ be a Banach space that does not have finite cotype. Given $\varepsilon>0$, for every finite set $\left\{x_{1}, \ldots, x_{n}\right\} \subset X$ there exists $Q \in \mathcal{M}(Z, S)$ such that

$$
\left\|T x_{i}\right\| \leq(1+\varepsilon)\left\|Q x_{i}\right\|
$$

for $i=1, \ldots, n$ and all $T \in \mathcal{M}(Z, S)$.

Proof. Since $Y$ does not have finite cotype, $Y$ contains $\ell_{n}^{\infty}$ uniformly $\left(\ell_{n}^{\infty}=\right.$ $\left.\left(\mathbb{R}^{n},\|\cdot\|_{\infty}\right)\right)$. By [2, theorem 14.1], for every $\varepsilon>0$ and $n \in \mathbb{N}$, there is an isomorphism $J_{n}$ from $\ell_{n}^{\infty}$ onto a subspace of $Y$ satisfying $\left\|J_{n}^{-1}\right\|=1$ and $\left\|J_{n}\right\| \leq 1+\varepsilon$ for all $n \in \mathbb{N}$.

Given $\left\{x_{1}, \ldots, x_{n}\right\} \subset X$, choose $z_{i}^{*} \in B_{Z^{*}}$ so that $\left|z_{i}^{*}\left(S x_{i}\right)\right|=\left\|S x_{i}\right\|$ for $i=$ $1, \ldots, n$. Put $y_{i}=J_{n} e_{i},\left(e_{i}\right)_{i=1}^{n}$ being the unit basis of $\ell_{n}^{\infty}$. We define an operator $Q: X \longrightarrow Y$ by

Then we have:

$$
Q x=\frac{1}{1+\varepsilon} J_{n}\left(\left(\left\langle S x, z_{i}^{*}\right\rangle\right)_{1}^{n}\right) .
$$

$$
\|Q x\| \leq(1+\varepsilon)^{-1}\left\|J_{n}\right\|\left\|\left(\left\langle S x, z_{i}^{*}\right\rangle\right)_{1}^{n}\right\|_{\infty} \leq\|S x\| \sup _{i}\left\|z_{i}^{*}\right\| \leq\|S x\|
$$

and this proves that $Q \in \mathcal{M}(Z, S)$.

Finally, we need to prove that $\left\|T x_{i}\right\| \leq(1+\varepsilon)\left\|Q x_{i}\right\|$ for $i=1, \ldots, n$ and all $T \in \mathcal{M}(Z, S)$. Put $y_{i}^{*}=e_{i}^{*} \circ J_{n}^{-1},\left(e_{i}^{*}\right)_{i=1}^{n}$ being the unit basis of $\left(\ell_{n}^{\infty}\right)^{*} \simeq \ell_{n}^{1}$. Note that $\left\|y_{i}^{*}\right\| \leq 1$ for $i=1, \ldots, n$. We also denote by $y_{i}^{*}$ a Hahn-Banach extension of $e_{i}^{*} \circ J_{n}^{-1}$ to $Y$. It is easy to show that $\left\langle y_{i}, y_{j}^{*}\right\rangle=\delta_{i j}$. We have:

$$
\begin{aligned}
\left\|Q x_{j}\right\| & \geq\left|\left\langle Q x_{j}, y_{j}^{*}\right\rangle\right| \\
& =(1+\varepsilon)^{-1}\left|\sum_{i=1}^{n}\left\langle S x_{j}, z_{i}^{*}\right\rangle\left\langle y_{i}, y_{j}^{*}\right\rangle\right| \\
& =(1+\varepsilon)^{-1}\left|\left\langle S x_{j}, z_{j}^{*}\right\rangle\right| \\
& =(1+\varepsilon)^{-1}\left\|S x_{j}\right\| \\
& \geq(1+\varepsilon)^{-1}\left\|T x_{j}\right\|
\end{aligned}
$$

for all $T \in \mathcal{M}(Z, S)$ and $j=1, \ldots, n$.

Corollary 3.2. If the Banach space $Y$ does not have finite cotype, every equicompact subset of $\mathcal{K}(X, Y)$ may be uniformly dominated, on each finite subset of $X$, by a compact operator.

Now we give a partial converse of the last theorem in case $\mathcal{M} \subset \mathcal{K}(X, Y)$ is a SOTcountably compact set. Note that the absolutely convex hull of an equicompact set is equicompact, too. So, without loss of generality, we can suppose from now on that $\mathcal{M}$ is absolutely convex.

Theorem 3.3. Let $\mathcal{N}$ be an absolutely convex subset of $\mathcal{K}(X, Y)$ enjoying property $(F)$. If $\mathcal{M}$ is countably compact for the strong operator topology, then $\mathcal{M}$ is equicompact.

Proof. (a) First, we prove the theorem in case $X$ is a separable Banach space. Let $\left(x_{n}\right)_{n}$ be a dense sequence in $X$. By hypothesis, for every $n \in \mathbb{N}$, there exists $Q_{n} \in \mathcal{M}$ so that

$$
\left\|T x_{i}\right\| \leq C\left\|Q_{n} x_{i}\right\|
$$


for $i=1, \ldots, n$ and all $T \in \mathcal{M}$. The sequence $\left(Q_{n}\right)_{n}$ has a cluster point $Q \in \mathcal{M}$ for the SOT. Proceeding by contradiction, it is easy to show that

$$
\left\|T x_{i}\right\| \leq C\left\|Q x_{i}\right\|
$$

for $i=1, \ldots, n$ and all $T \in \mathcal{M}$. Now, given $x \in X$ and $\varepsilon>0$, choose $x_{i}$ such that $\left\|x-x_{i}\right\|<\varepsilon / K$, where $K=\sup _{T \in \mathcal{M}}\|T\|$. Using (11), we have

$$
\begin{aligned}
\|T x\| & \leq\left\|T x-T x_{i}\right\|+\left\|T x_{i}\right\| \\
& <\varepsilon+C\left\|Q x_{i}\right\| \\
& \leq \varepsilon+C\left(\left\|Q x_{i}-Q x\right\|+\|Q x\|\right) \\
& \leq \varepsilon+C \varepsilon+C\|Q x\|
\end{aligned}
$$

for all $T \in \mathcal{M}$. Letting $\varepsilon \rightarrow 0$ we conclude $\|T x\| \leq C\|Q x\|$ for all $T \in \mathcal{M}$.

(b) Consider now an arbitrary Banach space $X$. In order to show that $\mathcal{M}$ is equicompact, we will prove that $\mathcal{M}$ has property $(P)$. Let $\left(x_{n}\right)_{n}$ be a bounded sequence in $X$ and put $H=\overline{\operatorname{span}}\left\{x_{n}: n \in \mathbb{N}\right\}$. If $i_{H}$ denotes the inclusion map from $H$ into $X$, then part (a) applied to the set $\mathcal{N}=\left\{T \circ i_{H}: T \in \mathcal{M}\right\} \subset \mathcal{K}(H, Y)$ yields the equicompactness of $\mathcal{N}$ and, therefore, also of $\mathcal{M}$.

Examples. 1. A maximal equicompact set of operators valued in $\ell_{2}$ that fails property $(F)$. Let $\mathcal{M}$ be the set of all operators $T$ from $c_{0}$ into $\ell_{2}$ satisfying

$$
\|T \alpha\|_{2} \leq \sup _{n}\left|\frac{1}{\sqrt{n}} e_{n}^{*}(\alpha)\right|
$$

for all $\alpha \in c_{0}\left(\left(e_{n}^{*}\right)_{n}\right.$ denotes the unit basis of $\left.\ell^{1}\right)$. By contradiction, suppose there exists a positive constant $C$ such that, for every finite set $\left\{\alpha_{1}, \ldots, \alpha_{n}\right\} \subset c_{0}$, there is an operator $Q \in \mathcal{M}$ such that $\left\|T \alpha_{k}\right\|_{2} \leq C\left\|Q \alpha_{k}\right\|_{2}$ for $k=1, \ldots, n$ and all $T \in \mathcal{M}$. In particular, for every $n \in \mathbb{N}$, there exists $Q_{n} \in \mathcal{M}$ so that

$$
\left\|T e_{k}\right\|_{2} \leq C\left\|Q_{n} e_{k}\right\|_{2}
$$

for $k=1, \ldots, n$ and all $T \in \mathcal{M}$ (here $\left(e_{n}\right)_{n}$ denotes the unit basis of $c_{0}$ ). Then we have

$$
\sum_{k=1}^{n}\left\|T_{k} e_{k}\right\|_{2}^{2} \leq C^{2} \sum_{k=1}^{n}\left\|Q_{n} e_{k}\right\|_{2}^{2}
$$

for all $n \in \mathbb{N}$ and all $\left\{T_{1}, \ldots, T_{n}\right\} \subset \mathcal{M}$. Now we consider the operators $T_{k} \in \mathcal{M}$ defined by $T_{k} \alpha=\frac{1}{\sqrt{k}} e_{k}^{*}(\alpha) u_{k}$ for all $\alpha \in c_{0}$, where $\left(u_{n}\right)_{n}$ is the unit basis of $\ell_{2}$. Notice that $\left\|T_{k} e_{k}\right\|_{2}=\frac{1}{\sqrt{k}}$; then (2) yields

$$
C^{-2} \sum_{k=1}^{n} \frac{1}{k} \leq \sum_{k=1}^{n}\left\|Q_{n} e_{k}\right\|_{2}^{2}
$$

for all $n \in \mathbb{N}$. Finally, recall that every bounded operator $T$ from $c_{0}$ into $\ell_{2}$ is 2-summing and $\pi_{2}(T) \leq \lambda\|T\|$, for some constant $\lambda>0$ (see [2, theorem 3.5]). This fact allows us to obtain

$$
\pi_{2}\left(Q_{n}\right)^{2} \geq \sum_{k=1}^{n}\left\|Q_{n} e_{k}\right\|_{2}^{2} \geq C^{-2} \sum_{k=1}^{n} \frac{1}{k}
$$

for all $n \in \mathbb{N}$. This is a contradiction because $\mathcal{M}$ is bounded for the $\pi_{2}$-norm.

2. It is interesting to show that the countable compactness of $\mathcal{M}$ cannot be omitted in Theorem [3.3. For example, consider the closed unit ball of $\mathcal{L}\left(c_{0}, c_{0}\right)$. 
This ball is the same that $\mathcal{N}\left(c_{0}, I\right), I$ being the identity map on $c_{0}$. The proof of Theorem 3.1 shows that $\mathcal{M}\left(c_{0}, I\right)$ has the following property:

"There exists a positive constant $C$ such that, for every finite set

$\left\{\alpha_{1}, \ldots, \alpha_{n}\right\} \subset c_{0}$, there is an operator of finite rank $Q \in \mathcal{M}\left(c_{0}, I\right)$

satisfying $\left\|T \alpha_{k}\right\| \leq C\left\|Q \alpha_{k}\right\|$ for $k=1, \ldots, n$ and all $T \in \mathcal{M}\left(c_{0}, I\right)$."

This implies that the closed unit ball of $\mathcal{K}\left(c_{0}, c_{0}\right), \mathcal{N}$, has property $(F)$. Nevertheless, we are going to show that $\mathcal{M}$ is not equicompact. For each $\delta=\left(\delta_{n}\right)_{n}$ belonging to the unit ball of $c_{0}$, we denote by $T_{\delta}$ the operator defined by $T_{\delta}\left(\alpha_{n}\right)_{n}=$ $\left(\alpha_{n} \cdot \delta_{n}\right)_{n}$ for all $\left(\alpha_{n}\right)_{n} \in c_{0}$. It is obvious that $T_{\delta} \in \mathcal{M}$ for all $\delta \in B_{c_{0}}$. For every $n \in \mathbb{N}$, we have $\left\|T_{e_{n}}\right\|=\left\|e_{n}\right\|=1$. Since $\left(e_{n}\right)_{n}$ is weakly null in $c_{0}$, this shows that $\mathcal{M}$ is not u.c.c.

\section{ACKNOWLEDGEMENT}

The authors thank the referee for his interesting suggestions.

\section{REFERENCES}

[1] N. Dunford, J. T. Schwartz, Linear operators. Part I: General theory, Wiley Interscience, New York and London, 1958. MR0117523(22:8302)

[2] J. Diestel, H. Jarchow, A. Tonge, Absolutely summing operators, Cambridge studies in advanced Mathematics 43, Cambridge University Press, Cambridge, 1995. MR.1342297 (96i:46001)

[3] F. Mayoral, Compact sets of compact operators in absence of $\ell^{1}$, Proc Amer. Math. Soc. 129 (2001), no. 1, 79-82. MR1784015 (2001e:46026)

[4] T. W. Palmer, Totally bounded sets of precompact operators, Proc. Amer. Math. Soc. 20 (1969), 101-106. MR0235425 (38:3734)

Departamento de Matemáticas, Facultad de Ciencias Experimentales, Campus Universitario del Carmen, Avda. de las Fuerzas Armadas s/n, 21071 Huelva, Spain

E-mail address: eserrano@uhu.es

Departamento de Matemáticas, Facultad de Ciencias Experimentales, Campus Universitario del Carmen, Avda. de las Fuerzas Armadas s/n, 21071 Huelva, Spain

E-mail address: candido@uhu.es

Departamento de Matemáticas, Facultad de Ciencias Experimentales, Campus Universitario del Carmen, Avda. de las Fuerzas Armadas s/n, 21071 Huelva, Spain

E-mail address: jmdelga@uhu.es 